\title{
ON IMPROVING THE CONVERGENCE RATE OF LINEAR CONTINUOUS-TIME SYSTEMS SUBJECT TO ASYMMETRICALLY CONSTRAINED CONTROL
}

\author{
A. BADDOU, M. NAIB, F. TADEO, AND A. BENZAOUIA
}

Received 5 April 2005; Revised 9 March 2006; Accepted 4 April 2006

This paper solves the problem of controlling linear continuous-time systems subject to control signals constrained in magnitude (maybe asymmetrically). A controller design methodology is proposed, based on using an asymmetric Lyapunov function, that avoids the discontinuities in the control vector components resulting from the application of a piecewise linear control law previously proposed. The proposed method gives improved speed of convergence without discontinuities of the control vector components, respecting always the imposed asymmetric constraints. An example illustrates the approach.

Copyright (C 2006 A. Baddou et al. This is an open access article distributed under the Creative Commons Attribution License, which permits unrestricted use, distribution, and reproduction in any medium, provided the original work is properly cited.

\section{Introduction and notation}

Usually, real plants are subject to constrained variables. The most frequent constraints are of saturation type, that is, limitations on the magnitude of certain variables, usually the control signal. Hence, the topic of designing control systems that maintain stability (and performance) in the presence of these constraints is a topic of continuing interest $[7,8,12]$. There are several approaches proposed in the literature to solve this problem; particularly successful is the positive invariance concept (see $[5,6]$ and references therein). In this approach a dilemma appears between the size of the domain of admissible initial states and the speed of the asymptotic convergence [1].

Recently, this problem of amelioration of the convergence rate of systems with constraints on the control has been studied in $[4,9,10,13]$. For example, in [13] the problem is studied for linear systems with symmetrical constrained control using quadratic Lyapunov functions. In [4] the problem is studied for linear systems with asymmetric constraints on the control by using a piecewise linear control law computed with an asymmetric Lyapunov function. Unfortunately, in these previous works, the proposed control laws induce discontinuities on the control vector components. Effort has been made to smooth these discontinuities (see [9-11] and references therein). For example, 


\section{On the convergence rate of systems with constrained control}

in [9] the method developed in [13] is extended to obtain a controller that guarantees fulfilling the imposed symmetric constraints without any discontinuity on the control vector components.

The objective of this work is to improve the results of [4] by ensuring that the dynamics of the control evolve without any discontinuity during the evolution of the system. For this, only two state feedback regulators are calculated: a first regulator gain $F_{0}$ is computed such that a large domain of admissible initial states is obtained; then, a second regulator gain $F_{1}$ is computed to give good convergence performance. Using an asymmetrical Lyapunov function, a regulator gain $F$ is calculated evolving smoothly from the initial value $F_{0}$ to the final value $F_{1}$. The resulting control law gives a continuous control vector respecting the asymmetric constraints without any saturation. The comparison between the obtained results and the previous results in [4], using the same example, shows the interest of this method.

The notation used in this work is as follows.

(i) For vectors $x, y \in \mathbb{R}^{n}$,

(1) $x \leq y$ if and only if $x_{i} \leq y_{i}, i=1, \ldots, n$;

(2) $x<y$ if and only if $x_{i}<y_{i}, i=1, \ldots, n$;

(3) $x_{i}^{+}=\sup _{i}\left(x_{i}, 0\right)$;

(4) $x_{i}^{-}=\sup _{i}\left(-x_{i}, 0\right)$.

(ii) For two subsets $S_{1}, S_{2} \subset \mathbb{R}^{m}, S_{1} \backslash S_{2}=\left\{x \in \mathbb{R}^{m} \mid x \in S_{1}\right.$ and $\left.x \notin S_{2}\right\}$.

(iii) For a matrix $H \in \mathbb{R}^{m \times m}$,

$$
\begin{gathered}
\tilde{H}=\left[\begin{array}{ll}
H_{1} & H_{2} \\
H_{2} & H_{1}
\end{array}\right] \in \mathbb{R}^{2 m \times 2 m}, \\
H_{1}(i, j)=\left\{\begin{array}{ll}
h_{i j} & \text { if } i=j, \\
h_{i j}^{+} & \text {if } i \neq j,
\end{array} H_{2}(i, j)=\left\{\begin{array}{ll}
0 & \text { if } i=j, \\
h_{i j}^{-} & \text {if } i \neq j,
\end{array} \quad 1 \leq i \leq m, 1 \leq j \leq m .\right.\right.
\end{gathered}
$$

(iv) $\rrbracket_{m}$ is the identity matrix of $\mathbb{R}^{m}$.

The paper is organized as follows: preliminaries are given in Section 2. Section 3 presents the main results and an illustrative example. Finally, some conclusions are given.

\section{Preliminaries}

In this section, the studied problem is presented together with the preliminary results on which this work is based. For this, consider the following linear continuous-time system:

$$
\dot{x}=A x+B u,
$$

where $x \in \mathbb{R}^{n}$ is the state vector and $u \in \mathbb{R}^{m}$ is the control vector. We assume that (2.1) is stabilizable and that matrix $A$ possesses at least $n-m$ stable eigenvalues. The control vector $u$ is subject to linear constraints of the form $u \in \Omega \subset \mathbb{R}^{m}$, with

$$
\Omega=\left\{u \in \mathbb{R}^{m} \mid-u_{\min } \leq u \leq u_{\max }\right\},
$$

where $u_{\max }$ and $u_{\min }$ are given constant vectors in $\mathbb{R}^{m}$ with positive components. 
A. Baddou et al. 3

Now, consider the state feedback stabilizing control law

$$
u(t)=F_{0} x(t)
$$

The control is admissible (i.e., $u \in \Omega$ ) only if the trajectory of the state remains inside the polyhedral domain $\mathscr{D}_{0}$, defined as

$$
\mathscr{D}_{0}=\mathscr{D}\left(F_{0}, u_{\min }, u_{\max }\right)=\left\{x \in \mathbb{R}^{n} \mid-u_{\min } \leq F_{0} x \leq u_{\max }\right\} .
$$

In order to ensure the admissibility of the control, we must then guarantee that the state does not leave the domain $\mathscr{D}_{0}$ during the system evolution.

Definition 2.1. A subset $\mathscr{D}$ of $\mathbb{R}^{n}$ is said to be positively invariant with respect to a given system if every trajectory of the system starting inside $\mathscr{D}$ does not leave $\mathscr{D}$.

Taking into account (2.3), system (2.1) becomes

$$
\dot{x}=\left(A+B F_{0}\right) x
$$

which represents the stable system in closed loop (i.e., matrix $A+B F_{0}$ is Hurwitz) if the saturations do not occur. Consider the transformation $z=F_{0} x$, then $\dot{z}=F_{0}\left(A+B F_{0}\right) x$. If there exists a matrix $H_{0}$ such that $F_{0}\left(A+B F_{0}\right)=H_{0} F_{0}$, then system (2.5) and domain (2.4) become, respectively,

$$
\begin{gathered}
\dot{z}=H_{0} z, \\
\mathscr{D}\left(\mathbb{\square}_{m}, u_{\min }, u_{\max }\right)=\left\{z \in \mathbb{R}^{m} \mid-u_{\min } \leq z \leq u_{\max }\right\} .
\end{gathered}
$$

Theorem 2.2 [5]. The function

$$
v(z)=\max _{i} \max \left(\frac{z_{i}^{+}}{u_{\max }^{i}}, \frac{z_{i}^{-}}{u_{\min }^{i}}\right)
$$

with $u_{\max }>0$ and $u_{\min }>0$, which is continuous positive definite, is a Lyapunov function of (2.6), and $\mathscr{D}\left(\square_{m}, u_{\min }, u_{\max }\right)=\left\{z \in \mathbb{R}^{m} \mid v(z) \leq 1\right\}$ is a stability domain of the system (2.6) if and only if

$$
\widetilde{H_{0}} U \leq 0
$$

where

$$
U=\left[\begin{array}{l}
u_{\max } \\
u_{\min }
\end{array}\right] \in \mathbb{R}^{2 m} .
$$

In order to apply this result to the system (2.1) fulfilling (2.2) and (2.3), one must look for a couple of matrices $\left(F_{0}, H_{0}\right)$ such that the two following relations are satisfied:

$$
\begin{gathered}
F_{0} A+F_{0} B F_{0}=H_{0} F_{0}, \\
\widetilde{H_{0}} U \leq 0 .
\end{gathered}
$$


4 On the convergence rate of systems with constrained control

Following the steps of the controller design method in [4], a diagonal matrix $H_{0}$ is chosen such that

$$
\widetilde{H_{0}} U<-\varepsilon U,
$$

where $\varepsilon$ is a positive real scalar. The gain matrix $F_{0}$ is obtained from the resolution of the equation

$$
X A+X B X=H_{0} X
$$

One can refer to [2-4] for the resolution of this equation. From (2.11), the obtained regulator ensures that domain (2.4) is positively invariant with respect to system (2.5) (see [5]).

The control law proposed in [4] uses a gain scheduling approach where $N$ different controller gains are used. The parameter $N$ is chosen a priori and the rate of decreasing Lyapunov function is given by

$$
1<\alpha_{0}<\exp \left(\frac{1}{N} \min _{j \in J} \log \left(\frac{\left(\widetilde{F_{0} B} U\right)_{j}+\left(\widetilde{H_{0}} U\right)_{j}}{\left(\widetilde{F_{0} B} U\right)_{j}+\varepsilon U_{j}}\right)\right)
$$

Using this expression, the remaining regulator gains are obtained from the following equation:

$$
F_{i}=\alpha_{0}^{i} F_{0}, \quad i=1, \ldots, N
$$

The corresponding matrices $H_{i}$, satisfying $F_{i} A+F_{i} B F_{i}=H_{i} F_{i}$, are given by

$$
H_{i}=H_{0}+\left(\alpha_{0}^{i}-1\right) F_{0} B
$$

and the inequality

$$
\widetilde{H}_{i} U<-\varepsilon \alpha_{0}^{i} U
$$

is satisfied for $i=1, \ldots, N$.

Then, the applied control law is given by

$$
u(t)=F_{i} x(t)
$$

which is switched from the gain $F_{i-1}$ to the gain $F_{i}$ when the state crosses inside the boundaries of the polyhedral domain $D\left(F_{i}, u_{\min }, u_{\max }\right), i=1, \ldots, N$. Then, the resulting control vector experiences $N$ discontinuities.

In order to overcome the drawback of discontinuities in the control vector, the methodology proposed in this paper considers a feedback law of only one switch because the obtained results can be extended to smoothen the discontinuity of a controller with 
multiple switches. Since the proposed continuous law guarantees a progressively increasing convergence rate, only the outermost and the innermost polyhedral domains $\mathscr{D}_{0}$ and $\mathscr{D}_{1}$, corresponding to the feedback gains $F_{0}$ and $F_{1}$, are used. The control law starts with a regulator gain $F_{0}$ which permits to benefit from a large set of admissible initial states $\mathscr{D}_{0}$ and finishes with a regulator gain $F_{1}$, which permits to benefit from good convergence rate performance. Because of the dilemma between the size of the set of admissible states and the good convergence performance, the closed-loop system obtained with $F_{0}$ usually gives bad convergence performance while the set of admissible states $\mathscr{D}_{1}$ is of a small size.

To derive the regulator gains, it is assumed that the regulator gain $F_{1}$ can be obtained from $F_{0}$ as follows:

$$
F_{1}=\mu F_{0}
$$

where $\mu>1$.

The link between $\mu$ in this work and $\alpha_{0}$ in [4] is that

$$
\mu=\alpha_{o}^{N}
$$

It is easy to see that $\mathscr{D}_{1} \subset \mathscr{D}_{0}: x \in \mathscr{D}_{1}$ implies that $-u_{\min } \leq F_{1} x \leq u_{\max }$, or equivalently $-u_{\min } \leq \mu F_{0} x \leq u_{\max }$. Dividing by $\mu$ and taking into account that $\mu>1$ gives the result.

It is also easy to see that the corresponding matrix $H_{1}$ can be obtained from $H_{0}$ as follows:

$$
H_{1}=H_{0}+(\mu-1) F_{0} B
$$

To show this, consider the two equations

$$
\begin{aligned}
& F_{1} A+F_{1} B F_{1}=H_{1} F_{1}, \\
& F_{0} A+F_{0} B F_{0}=H_{0} F_{0},
\end{aligned}
$$

which are equivalent to

$$
\begin{gathered}
F_{0} A+\mu F_{0} B F_{0}=H_{1} F_{0}, \\
F_{0} A+F_{0} B F_{0}=H_{0} F_{0} .
\end{gathered}
$$

Subtracting the second equation from the first and using the fact that the obtained solution of (2.13) is of full rank gives the result.

Until now, matrices $H_{0}, H_{1}, F_{0}$, and $F_{1}$ are obtained. Furthermore, from (2.12) the positive invariance property of $\mathscr{D}_{0}$ is obtained. Since parameter $\mu$ is chosen such that

$$
\widetilde{H_{1}} U<-\epsilon \mu U
$$

the positive invariance property of domain $\mathscr{D}_{1}$ is also guaranteed. 
6 On the convergence rate of systems with constrained control

\section{Main results}

In this section, the main results of this paper are presented. The idea consists in proposing a different technique to compute the rate $\alpha$, which is now considered time-varying in such a way that the discontinuity of the piecewise controller proposed in the previous work is avoided.

Based on the previous development, this work can be accomplished in two steps: from the reasoning developed in the introduction, the first step is completed. It consists in finding $H_{0}, \varepsilon, F_{0}, H_{1}, \mu$, and $F_{1}$ satisfying all the necessary requirements. In the second step, starting with the regulator gain $F_{0}$, the control gain evolves continuously until finishing the regulator gain $F_{1}$. That is, at each time, the regulator must depend upon the state in the following way:

$$
F=\alpha(x(t)) F_{0}
$$

where

$$
1 \leq \alpha(x(t)) \leq \mu
$$

Using the control law

$$
u(t)=F x(t)
$$

with (3.1), the closed-loop system is given by

$$
\dot{x}(t)=(A+B F) x(t)=\left(A+\alpha(x(t)) B F_{0}\right) x(t)=A_{c}(x) x(t) .
$$

Recall that the objective is to make domain $\mathscr{D}=\mathscr{D}\left(F, u_{\min }, u_{\max }\right)$ positively invariant at any time. Thus, one has to look for a matrix $H$ satisfying the following two relations at any time:

$$
\begin{gathered}
F A+F B F=H F, \\
\tilde{H} U \leq 0 .
\end{gathered}
$$

As in (2.21), matrix $H$ can be obtained as follows:

$$
H=H_{0}+(\alpha(x(t))-1) F_{0} B
$$

LEMMA 3.1. If matrix $H$ is obtained from (3.6) such that relations (2.12) and (2.24) are satisfied, then $\tilde{H} U<0$ for all $t$.

Proof. Since $H_{0}$ is a given diagonal matrix, according to (2.12), one can write

$$
\widetilde{H}=\widetilde{H_{0}}+(\alpha(x(t))-1) \widetilde{F_{0} B}
$$


If $\widetilde{F_{0} B} U \leq-\varepsilon U$, then the result holds for every $\alpha$. Otherwise, there exists a set of indices $J \subset[1, \ldots, 2 m]$ such that $\left(\widetilde{F_{0} B} U\right)_{i}>-\varepsilon U_{i}$ for $i \in J$. In this case, by virtue of $(2.21)$ and (2.24), one can write

$$
\widetilde{H_{1}} U=\widetilde{H_{0}} U+(\mu-1) \widetilde{F_{0} B} U<-\epsilon \mu U
$$

This implies that

$$
\mu\left(\widetilde{F_{0} B} U+\epsilon U\right)<\widetilde{F_{0} B} U-\widetilde{H_{0}} U
$$

Then $\mu$ satisfies the following inequality:

$$
\mu<\min _{i \in J}\left(\frac{\left(\widetilde{F_{0} B} U\right)_{i}-\left(\widetilde{H_{0}} U\right)_{i}}{\left(\widetilde{F_{0} B} U\right)_{i}+\varepsilon U_{i}}\right) .
$$

Taking account of (3.2) one can write

$$
\alpha<\min _{i \in J}\left(\frac{\left(\widetilde{F_{0} B} U\right)_{i}-\left(\widetilde{H_{0}} U\right)_{i}}{\left(\widetilde{F_{0} B} U\right)_{i}+\varepsilon U_{i}}\right) .
$$

Hence, the last inequality can also equivalently be written as follows:

$$
\widetilde{H_{0}} U+(\alpha-1) \widetilde{F_{0} B} U<-\epsilon \mu U
$$

Taking into account (3.7), one obtains

$$
\tilde{H} U<-\varepsilon \alpha U<0 \text {. }
$$

Thus, the result is proved.

Remark 3.2. For $F_{0}$ solution of (2.13) with $H_{0}$ satisfying (2.12), from relations (2.19) and (3.1), the obtained feedback gain matrices $F_{1}=\mu F_{0}$ and $F=\alpha(x) F_{0}$ have the same kernel $\mathscr{K}$, which is spanned by the eigenvectors associated to the stable eigenvalues of the openloop system. Consequently, all the obtained domains are nested and domain $\mathscr{D}_{1}$ is the smallest set such that $\mathscr{K} \subset \mathscr{D}_{1}$ (see [4]).

Consider the following function:

$$
w(x)=\max _{i} \max \left(\frac{\left(F_{0} x\right)_{i}^{+}}{u_{\max }^{i}}, \frac{\left(F_{0} x\right)_{i}^{-}}{u_{\min }^{i}}\right),
$$

and the subset $\mathscr{Y}=\mathscr{D}_{0} \backslash \mathscr{D}_{1}$. 
8 On the convergence rate of systems with constrained control

Lemma 3.3. Function $w(x)$ given by (3.14) is a Lyapunov function of system (2.1) with (2.24), (3.2), and (3.3) on the subset $\mathscr{Y}$, with matrix $F_{0}$ solution of (2.13) and matrix $H_{0}$ given according to (2.12).

Proof. It is clear that $w(x)$ is a positive definite function on the set $\mathscr{S}$. Furthermore, $w(x)>0$ for all $x \in S$ because $\mathscr{Y}$ does not contain the kernel of matrix $F_{0}$. To complete the proof, we must show that function $w(x)$ is decreasing along the trajectories of the system. Following [5], we compute the directional derivative of $w(x)$, that is,

$$
\dot{w}(x)=\frac{d w(x)}{d t}=\lim _{h \rightarrow 0^{+}} \frac{1}{h}\left[w\left(x+h A_{c}(x) x\right)-w(x)\right] .
$$

Substituting function $w(x)$ by its expression (3.14), it follows that

$$
\dot{w}(x)=\lim _{h \rightarrow 0^{+}} \frac{1}{h}\left[\max _{i} \max \left(\frac{\left[F_{0}\left(x+h A_{c}(x) x\right)\right]_{i}^{+}}{u_{\max }^{i}}, \frac{\left[F_{0}\left(x+h A_{c}(x) x\right)\right]_{i}^{-}}{u_{\min }^{i}}\right)-w(x)\right] .
$$

Recall that matrix $H$ satisfies (3.6). Then one can obtain that

$$
\begin{aligned}
F_{0}(A+B F) & =F_{0} A+\alpha F_{0} B F_{0} \\
& =F_{0} A+F_{0} B F_{0}+(\alpha-1) F_{0} B F_{0} \\
& =H_{0} F_{0}+(\alpha-1) F_{0} B F_{0} \\
& =\left(H_{0}+(\alpha-1) F_{0} B\right) F_{0} \\
& =H F_{0} .
\end{aligned}
$$

Equation (3.17) leads to the following:

$$
F_{0}\left[x+h A_{c}(x) x\right]=\left(F_{0}+h F_{0} A+h F_{0} B F\right) x=\left(F_{0}+h H F_{0}\right) x=\left(\square_{m}+h H\right) F_{0} x .
$$

Denoting $C=\rrbracket_{m}+h H$, then

$$
\dot{w}(x)=\lim _{h \rightarrow 0^{+}} \frac{1}{h}\left[\max _{i} \max \left(\frac{\left(C F_{0} x\right)_{i}^{+}}{u_{\max }^{i}}, \frac{\left(C F_{0} x\right)_{i}^{-}}{u_{\min }^{i}}\right)-w(x)\right] .
$$

On the other hand, if (3.13) is satisfied, one can write

$$
-u_{\min } \leq F x \leq u_{\max } .
$$


Taking account of (3.1) and (3.2), these inequalities imply that

$$
-u_{\min } \leq F_{0} x \leq u_{\max }
$$

Following the same steps as in [5], one obtains that

$$
\dot{w}(x) \leq \max _{i} \max \left[\frac{\left(H_{1} u_{\max }+H_{2} u_{\min }\right)_{i}}{u_{\max }^{i}} w(x), \frac{\left(H_{2} u_{\max }+H_{1} u_{\min }\right)_{i}}{u_{\min }^{i}} w(x)\right] .
$$

By virtue of (3.13), it follows that

$$
\begin{aligned}
& H_{1} u_{\max }+H_{2} u_{\text {min }} \leq-\varepsilon \alpha u_{\max }, \\
& H_{2} u_{\max }+H_{1} u_{\text {min }} \leq-\varepsilon \alpha u_{\min },
\end{aligned}
$$

which leads to

$$
\dot{w}(x) \leq-\varepsilon \alpha w(x)<0 .
$$

The proof is then completed.

Since the main objective remains to smoothen the discontinuities on the control, one can impose that the control switches continuously. Thus, the proposed idea is to compute the gain feedback matrices from the following lemma.

Lemma 3.4. Consider system (2.1) with $H_{0}$ satisfying (2.12) and $H_{1}$ satisfying (2.24), if the control law is given by (3.1) with the scalar $\alpha(t)$ chosen as follows:

$$
\alpha(x(t))=\frac{1}{w(x(t))},
$$

then

(i) the scalar $\alpha(t)$ is strictly time increasing while $x \in \mathscr{Y}$,

(ii) the dynamics of the system are improved continuously through the nested domains,

(iii) the constraints on the control are respected with neither saturation nor discontinuity on the control vector components.

Proof. The first item follows from the fact that $w(x)$ is a Lyapunov function on $\mathscr{Y}$. The second item follows from the decreasing rate of $w(x)$. The third item is also true because $\tilde{H} U \leq-\varepsilon \alpha U$ according to the following: consider the transformation $z=F_{0} x$, then, $\dot{z}(t)=F_{0}(A+B F) x(t)$. Taking account of (3.17), the dynamic of the new system is given by $\dot{z}(t)=H x(t)$ while the set $\mathscr{D}_{0}$ becomes $\mathscr{D}_{z}=\mathscr{D}\left\{\mathbb{q}_{m}, u_{\min }, u_{\max }\right\}$. Condition $\tilde{H} U \leq 0$ is the necessary and sufficient condition for the set $\mathscr{D}_{z}$ to be positively invariant with respect to the new system. Since the scalar $\alpha \geq 1$, then the set $\mathscr{D}_{0}$ is positively invariant with respect to system in closed-loop (3.4), that is, the control is always admissible. 
From the previous lemmas, we can give the following result.

Theorem 3.5. System (2.1) is asymptotically stable using the control law (3.3) with (2.12), (2.24), (3.1), (3.14), and (3.25). Moreover, every trajectory starting from $x_{0} \in \mathscr{D}_{0}$ converges to the origin with neither saturation nor discontinuity of the control vector components.

Proof. Let $\mathscr{G}=\mathscr{D}_{1} \backslash \mathscr{K}$, then $\mathscr{D}_{0}$ can be decomposed as follows:

$$
\mathscr{D}_{0}=\mathscr{S} \bigcup \mathscr{G} \bigcup \mathscr{K}
$$

If the state is inside $\mathscr{S}$ then, from the Lyapunov function properties, the trajectory will converge to the set $\mathscr{G}$.

Remark 3.6. (1) From the previous development, the convergence rate $\alpha(x)$ increases continuously throughout the set $\mathscr{S}$ but remains always bounded by $\mu$. This coefficient is computed from (2.14), (2.20), and the necessary and sufficient condition (2.24) guaranteeing the positive invariance property of the set $\mathscr{D}_{1}$. However, from the proof of this result, if $\widetilde{F_{0} B} U \leq-\varepsilon U$, then $\mathscr{D}_{1}$ is positively invariant for every $\mu$, which leads to a free choice of the convergence rate. That is, $\mathscr{D}_{1}$ can be reduced to the kernel of $F_{0}$. Once matrix $F_{0}$ and the scalar $\mu$ are computed, the strategy to follow is to use the controller gain $F=\alpha(x) F_{0}$ until the scalar $\alpha(x)$ reaches $\mu$. Then, the gain switches to a static gain $F_{1}$, leading to the asymptotic stability of the system inside the set $\mathscr{D}_{1}$.

(2) From the expression of $w(x(t))$, suppose that the value of $w\left(x\left(t^{*}\right)\right)$ at the instant $t^{*}$ is obtained from the index $j$, that is,

$$
w\left(x\left(t^{*}\right)\right)=\frac{\left(F_{0} x\left(t^{*}\right)\right)_{j}}{q_{j}},
$$

where $q$ can take $u_{\min }$ or $u_{\max }$ as value. Then, the control vector at time $t^{*}$ is given by

$$
u\left(t^{*}\right)=\frac{1}{w\left(x\left(t^{*}\right)\right)} F_{0} x\left(t^{*}\right)
$$

The $j$ th component of $u\left(t^{*}\right)$ is equal to $q_{j}$, and this is true for every $t^{*}$. In conclusion, during the evolution of the system inside domain $\mathscr{Y}$, always one of the control components takes its maximal admissible value, improving the convergence of the process.

(3) The approach developed in [9] solves the problem for linear continuous-time systems with symmetrical constraints on the control vector using quadratic Lyapunov functions. The approach presented here treats the asymmetrical case which is more convenient to the real applications, in this case, asymmetrical Lyapunov functions and the positive invariance concept are used. 
All the steps of the proposed methodology are summarized in the following algorithm. Algorithm 3.7.

Step 1 . Verify that the system matrix $A$ possesses at least $(n-m)$ stable eigenvalues.

Step 2. Choose matrix $H_{0}$ and $\varepsilon$ such that (2.12) is satisfied; and solve (2.13) to obtain $F_{0}$. Step 3. Compute $\mu$ according to (2.14), (2.20), and (2.24) (see [4] for more details).

Step 4. Start the closed-loop system with the regulator gain $F_{0}$ and change it to $F=\alpha(x) F_{0}$ according to (3.25) while the state is inside the set $\mathscr{Y}$. When $\alpha$ reaches $\mu$, the controller gain becomes $F_{1}=\mu F_{0}$ leading to the asymptotic stability of the system inside the set $\mathscr{D}_{1}$.

In order to illustrate the different properties of the obtained controller with the proposed method, we consider the example studied in [4] in order to compare the results of the two controller designs.

Example 3.8. Consider a continuous-time system given by (2.1) with the following data:

$$
\begin{gathered}
A=\left[\begin{array}{ccc}
1 & -2 & -3 \\
0.45 & -4 & 4 \\
2 & -0.9 & 15
\end{array}\right], \quad B=\left[\begin{array}{cc}
1 & 0.2 \\
0.3 & -1 \\
0 & -0.5
\end{array}\right], \\
u_{\min }=\left[\begin{array}{ll}
7.1 & 8
\end{array}\right]^{T}, \quad u_{\max }=\left[\begin{array}{ll}
4 & 1
\end{array}\right]^{T}, \quad U=\left[\begin{array}{llll}
4 & 1 & 7.1 & 8
\end{array}\right]^{T} .
\end{gathered}
$$

In this case $m=2$ and $n=3$, and the spectrum of the system matrix $A$ is

$$
\sigma(A)=\{1.5046,14.2937,-3.7983\}
$$

This system possesses one stable eigenvalue (because $n-m=1$ ). As in [4], we choose the assigned spectrum of the system in closed loop to be

$$
\wedge=\left\{\begin{array}{lll}
-3.7983 & -1 & -1.1
\end{array}\right\} .
$$

Solving (2.13) and taking $\varepsilon=0.2$ and $N=3$, we obtain $\alpha_{0}=1.114$. This means that, in the proposed method, the corresponding scalar $\mu=(1.114)^{3}$.

Figure 3.1 plots the components of the state and the control vectors for the following three situations:

(1) using just $F_{0}$ without any change of regulator;

(2) using the controller designed in [4];

(3) using the controller of the proposed method. 
12 On the convergence rate of systems with constrained control

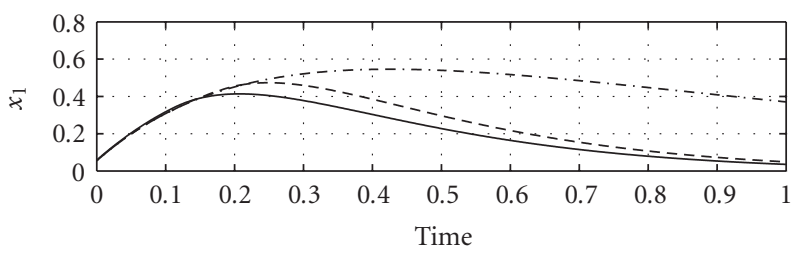

(a)

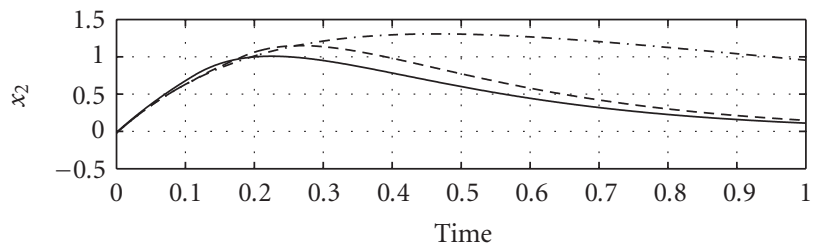

(b)

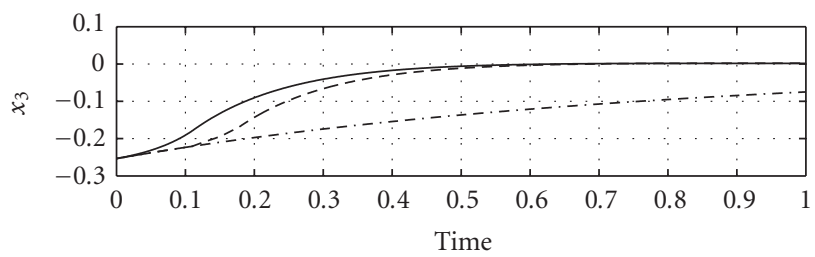

(c)

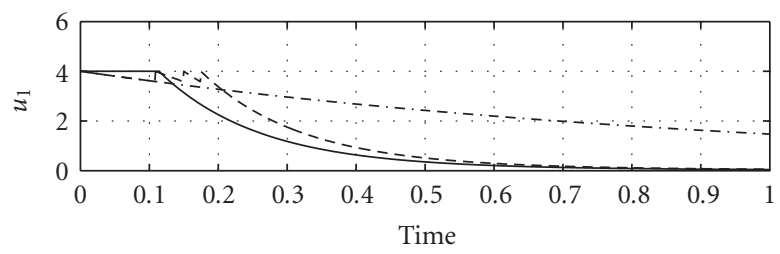

(d)

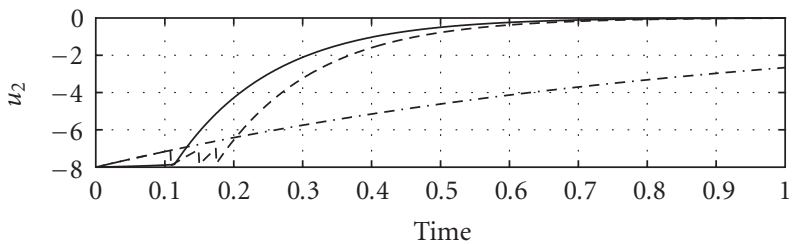

-. Trajectory with initial gain

- - - Trajectory with discrete switching

— Trajectory with continuous switching

(e)

Figure 3.1. Evolution of states and controls. 
It is clear that with the proposed method, the convergence rate of the closed-loop system is improved with neither saturation nor discontinuity on the components of the control vector. Furthermore, the first component of the control vector takes $\left(u_{\max }\right)_{1}=4$ during the evolution of the process inside $\mathscr{S}$, according to the second item of Remark 3.6.

\section{Conclusion}

In this work, a new controller design methodology has been proposed to improve the convergence rate of a linear continuous-time system with asymmetric constraints on its control vector. The obtained regulator also permits to benefit from a large domain of admissible state and avoids discontinuities and saturations of the control vector. An illustrative example shows the improvement obtained with the proposed method, compared with previous approaches.

\section{Acknowledgments}

This work is the product of collaboration between University Cadi Ayyad of Morocco and University of Valladolid of Spain funded by MAE-AECI under Grant 08P/04 and CICYT under Grant DPI2004-07444-C04-02.

\section{References}

[1] A. Baddou and A. Benzaouia, On the dynamic improvement in linear constrained control discretetime systems, International Journal of Systems Sciences 32 (2001), no. 4, 433-441.

[2] A. Benzaouia, The resolution of equation $X A+X B X=H X$ and the pole assignment problem, IEEE Transactions on Automatic Control 39 (1994), no. 10, 2091-2095.

[3] A. Benzaouia, M. Ait Rami, and S. El Faiz, Stabilization of linear systems with saturation: a Sylvester equation approach, IMA Journal of Mathematical Control and Information 21 (2004), no. 3, 247-259.

[4] A. Benzaouia and A. Baddou, Piecewise linear constrained control for continuous-time systems, IEEE Transactions on Automatic Control 44 (1999), no. 7, 1477-1481.

[5] A. Benzaouia and A. Hmamed, Regulator problem for linear continuous-time systems with nonsymmetrical constrained control, IEEE Transactions on Automatic Control 38 (1993), no. 10, 1556-1560.

[6] F. Blanchini, Set invariance in control, Automatica 35 (1999), no. 11, 1747-1767.

[7] Y. Eun, C. Gökçek, P. T. Kabamba, and S. M. Meerkov, An LQG approach to systems with saturating actuators and anti-windup implementation, Mathematical Problems in Engineering 8 (2002), no. $4-5,311-321$.

[8] P. O. Gutman and P. Hagander, A new design of constrained controllers for linear systems, IEEE Transactions on Automatic Control 30 (1985), no. 1, 22-33.

[9] T. Hu and Z. Lin, On improving the performance with bounded continuous feedback laws, IEEE Transactions on Automatic Control 47 (2002), no. 9, 1570-1575.

[10] T. Hu, Z. Lin, and Y. Shamash, On maximizing the convergence rate for linear systems with input saturation, IEEE Transactions on Automatic Control 48 (2003), no. 7, 1249-1253.

[11] R. Suárez, J. Álvarez-Ramírez, and J. Solís-Daun, Linear systems with bounded inputs: global stabilization with eigenvalue placement, International Journal of Robust and Nonlinear Control 7 (1997), no. 9, 835-845. 


\section{On the convergence rate of systems with constrained control}

[12] H. J. Sussmann, E. Sontag, and Y. Yang, A general result on stabilization of linear systems using bounded controls, IEEE Transactions on Automatic Control 39 (1994), no. 12, 2411-2425.

[13] G. F. Wredenhagen and P. R. Bélanger, Piecewise linear LQ control for systems with input constraints, Automatica 30 (1994), no. 3, 403-416.

A. Baddou: Département de Génie Industriel, Ecole Nationale des Sciences Appliquées, Ibn Zohr University, BP 1136, Agadir 80000, Morocco

E-mail address: baddou@ensa-agadir.ac.ma

M. Naib: Constrained and Robust Regulation Research Unit, Department of Physics, Faculty of Sciences Semlalia, Cadi Ayyad University, BP 2390, Marrakech, Morocco

E-mail address: m.naib@ucam.ac.ma

F. Tadeo: Departamento Ingeniería de Sistemas y Automática, Universidad de Valladolid, 47005 Valladolid, Spain

E-mail address: fernando@autom.uva.es

A. Benzaouia: Constrained and Robust Regulation Research Unit, Department of Physics, Faculty of Sciences Semlalia, Cadi Ayyad University, BP 2390, Marrakech, Morocco

E-mail address: benzaouia@ucam.ac.ma 


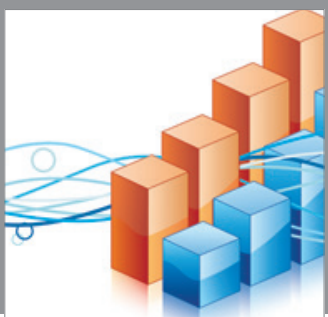

Advances in

Operations Research

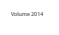

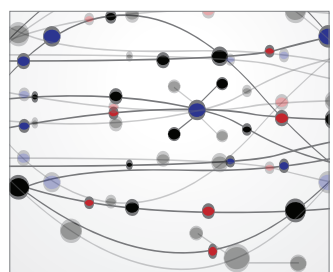

\section{The Scientific} World Journal
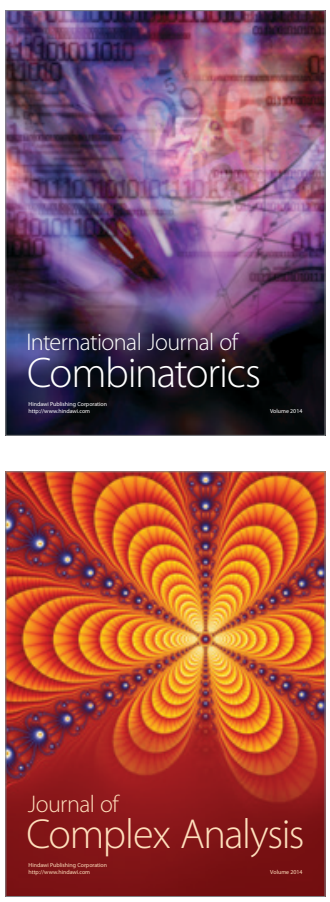

International Journal of

Mathematics and

Mathematical

Sciences
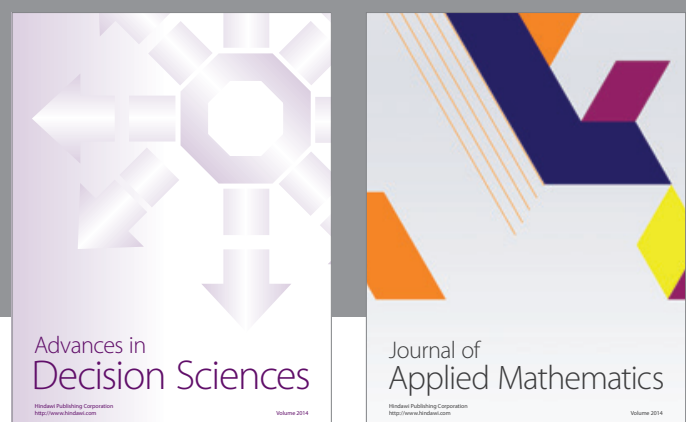

Journal of

Applied Mathematics
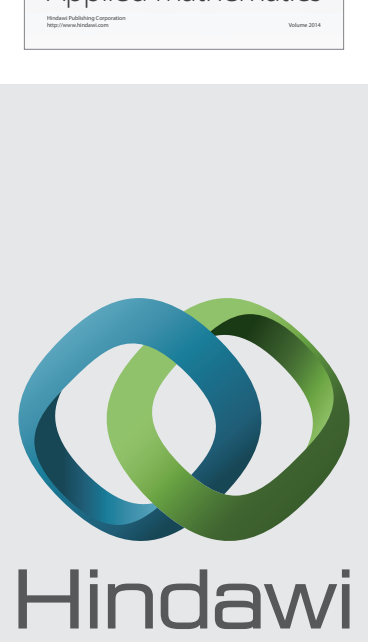

Submit your manuscripts at http://www.hindawi.com
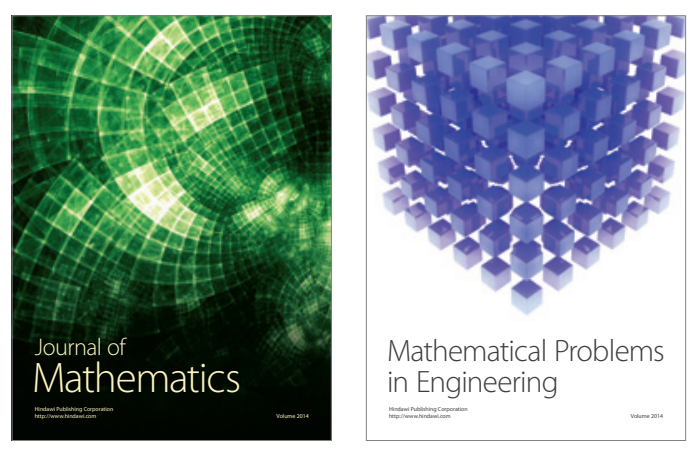

Mathematical Problems in Engineering
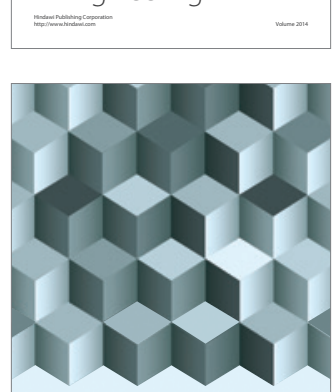

Journal of

Function Spaces
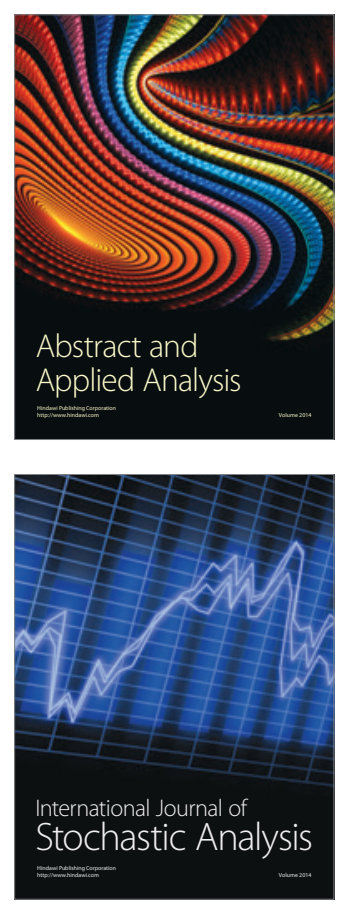

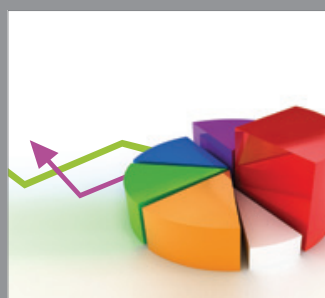

ournal of

Probability and Statistics

Promensencen
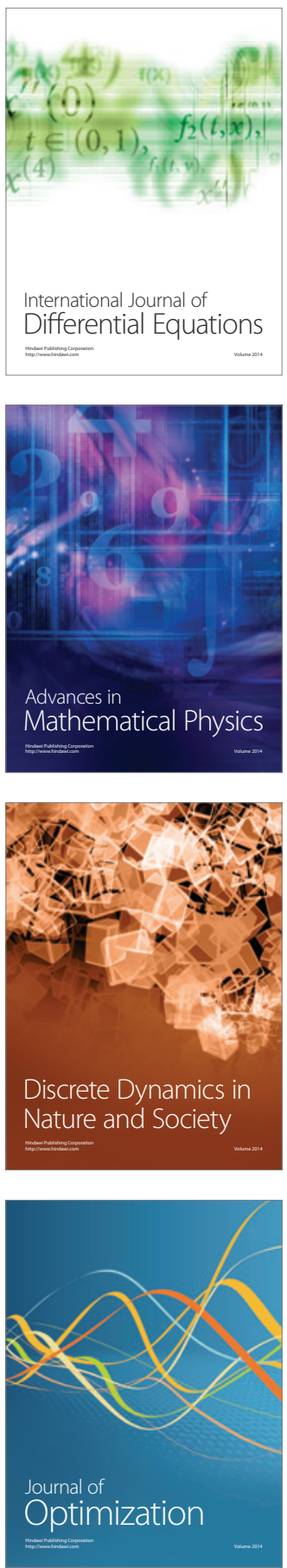